\title{
A Modular Antigen Presenting Peptide/Oligonucleotide Nanostructure Platform for Inducing Potent Immune Response
}

\author{
Sehmus Tohumeken, Nuray Gunduz, M. Burak Demircan, Gokhan Gunay, Ahmet E. Topal, \\ M. Aref Khalily, Turgay Tekinay, Aykutlu Dana, Mustafa O. Guler,* and Ayse B. Tekinay**
}

The design and development of vaccines, which can induce cellular immunity, particularly CD8+ T cells hold great importance since these cells play crucial roles against cancers and viral infections. Covalent conjugation of antigen and adjuvant molecules has been used for successful promotion of immunogenicity in subunit vaccines; however, the stimulation of the CD8+ T-cell responses by this approach has so far been limited. This study demonstrates a modular system based on noncovalent attachment of biotinylated antigen to a hybrid nanofiber system consisting of biotinylated self-assembling peptide and CpG oligodeoxynucleotides (ODN) molecules, via biotin-streptavidin interaction. These peptide/oligonucleotide hybrid nanosystems are capable of bypassing prior limitations related with inactivated or live-attenuated virus vaccines and achieve exceptionally high CD8+ T-cell responses. The nanostructures are found to trigger strong IgG response and effectively modulate cross-presentation of their antigen "cargo" through close proximity between the antigen and peptide/ODN adjuvant system. In addition, the biotinylated peptide nanofiber system is able to enhance antigen uptake and induce the maturation of antigen-presenting cells. Due to its versatility, biocompatibility, and biodegradability with a broad variety of streptavidin-linked antigens, the nanosystem shown here can be utilized as an efficient strategy for new vaccine development.

\section{Introduction}

Despite improvements in vaccine technology, effective vaccines are yet to be developed for diseases such as cancer, human immunodeficiency virus (HIV) infection, tuberculosis, and malaria, which together cause the deaths of more than 4 million people worldwide each year. ${ }^{[1]}$ It is also predicted that in 2030, cancers will claim the lives of more than 11 million people worldwide. ${ }^{[2]}$ Currently, clinically approved vaccine adjuvants such as aluminum hydroxide or the oil-in-water emulsion MF59 can promote humoral immune response, which is also called antibody mediated immunity, but fail to stimulate cellular immune response. ${ }^{[3]}$ Among cellular immune responses, the activation of CD8+ $\mathrm{T}$ cells is critical for the development of vaccines against cancer or intracellular pathogens such as HIV, malaria, and hepatitis $\mathrm{C}$ due to their ability to kill cancer and infected cells. As such, materials modulating both humoral and cellular responses in a synchronized manner are preferred in vaccine development. ${ }^{[4]}$ Accordingly, inactivated virus, live-attenuated virus, and viral vector-based subunit vaccines have been developed for inducing effective humoral and cellular responses. ${ }^{[5]}$ However, these approaches have so far faced problems involving incomplete viral inactivation, regain of virulence, and unfavorable host responses to viral vectors, which have limited their clinical utility. ${ }^{[6]}$

Safety concerns associated with inactivated and live-attenuated virus vaccines have led to the development of recombinant protein-based subunit vaccines, which consist of one or more recombinant proteins or polysaccharides and do not utilize the active viral agent in any form. However, subunit vaccines induce poor or nonexistent CD8+ T-cell responses and exhibit low cross-presentation efficiencies, as they are not uptaken, processed and/or presented to naive CD8+ T cells to the same extent as the entire viral unit. ${ }^{[7]}$ Antigens can be delivered in particulate form in order to generate specific T-cell responses. ${ }^{[8]}$ Encapsulated or conjugated antigens have likewise been shown to induce greater humoral responses than soluble antigens. ${ }^{[9]}$ Importantly, the cross-presentation and immunogenicity of antigens can be improved through the

DOI: 10.1002/adbi.201700015 
administration of immunostimulatory molecules or adjuvants including aluminum, MF59, MPL, AS04, AS01B, and AS02A, toll-like receptor (TLR) ligands, CpG-ODN1826, or oil-based adjuvants. ${ }^{10]}$ To induce an effective immune response, adjuvant and antigens should be in close proximity. ${ }^{[11]}$ The size of carrier materials is another important parameter for the generation of an effective immune response. Immunization with nanoparticles in the 200-600 $\mathrm{nm}$ range are known to induce higher levels of IFN- $\gamma$ production, upregulation of MHC class I molecules and the production of antibody isotypes favoring the Th1-type immune response. Immunization with 2-8 $\mu \mathrm{m}$ microparticles, in contrast, enhance IL-4 secretion, upregulate MHC class II expression, and direct the immune response toward the Th2-type. Moreover, nanoparticles in the 20-200 nm range can efficiently enter the lymphatic system through lymph drainage. ${ }^{[12]}$ Previous studies have also shown that epitope density displayed on the nanoparticles is crucial for effectively eliciting humoral immune responses, especially the IgG response. ${ }^{[13]}$ As such, materials to be used for antigen delivery applications should have controllable sizes and exhibit the ability to present epitopes efficiently to produce concerted T-cell responses. Highly effective T-cell vaccines fulfilling these criteria are still being sought, and a broad variety of methods have been employed for their development. ${ }^{[7 \mathrm{~b}, 12]}$

Electrostatic interaction mediated self-assembly methods have been used as a powerful strategy for the construction of functional materials which have explicit properties as ease of design with functional motifs, biocompatibility, and biodegradability, and therefore they exhibit immense potential for regenerative medicine, drug delivery, cell culture, analytic detection, biosensor, and immunotherapy applications. ${ }^{[14]}$ Selfassembled peptide nanostructures were previously shown as virus mimetic materials, powerful immune adjuvants, ${ }^{[15]}$ and an effective antigen delivery system, ${ }^{[14 \mathrm{~b}, 16]}$ which exhibit great potential to elicit strong immune responses while posing little to no health risks. Modification of these peptide-based nanostructures with antigens is important for better presentation of antigens to antigen presenting cells (APCs), however, these modifications are limited due to difficult chemical ligation steps. Covalent and noncovalent ligation methods are used for antigen and protein attachment to soft peptide-based nanostructures. Since the preparation of these materials via covalent conjugation is not readily applicable to all antigen fragments, noncovalent conjugation of an antigen or a protein was chosen as our strategy. The streptavidin-biotin system can be used in situations where direct covalent attachment is problematic, since the streptavidin-biotin interaction is among the strongest noncovalent interactions in nature. ${ }^{[17]}$ In a previous study, streptavidin was shown to bind strongly to biotinylated self-assembled nanostructures, and biotinylated insulin-like growth factor 1 was attached to bind to the peptide nanostructure through a streptavidin linker. ${ }^{[18]}$

In this work, we demonstrate a modular delivery system using a biotin-linked approach, which allows a broad diversity of antigens to be presented on the surface of peptide nanofibers. Nanostructures consisting of ODN1826 adjuvant (a TLR9 agonist) and biotinylated self-assembling peptide amphiphile (B-PA) allowed highly specific binding of antigens to their surface and enabled positioning of the antigens in close proximity with the adjuvant through the streptavidin linker. We therefore hypothesize that rationally designed nanofibrous complex made of B-PA can be used as an effective vaccine candidate to meet the stringent demands of the vaccine industry.

\section{Results and Discussion}

\subsection{Design and Characterization of the Nanofibrous Complex}

To obtain the self-assembled 1D antigen bearing nanofibrous complex (B-PA+oligodeoxynucleotide (ODN) + streptavidin + ovalbumin), B-PA, which allows binding of Ovalbumin (model antigen) via streptavidin was synthesized through solid phase peptide synthesis (Figure 1A and Figure S1 (Supporting Information)). The lauryl group conjugated to the peptide sequence drives self-assembly of these molecules through hydrophobic collapse in aqueous solutions. The Val-Val-Ala peptide sequence was used to facilitate $\beta$-sheet formation during selfassembly, and glycine was used as spacer. Two lysine residues were utilized in the synthesis of B-PA: one lysine was used to graft biotin to the peptide backbone while the second lysine was used to impart a positive charge to B-PA, which enhances its solubility in aqueous media. This configuration splits the B-PA molecule into two functional regions; a hydrophobic inner core (consisting of an alkyl tail and $\beta$-sheet forming residues) and a hydrophilic outer shell (consisting of bioactive sequences). ${ }^{[11,14 c, 19]}$ B-PA molecules and ODNs formed cylindrical nanostructures through electrostatic interactions.

Previously, we demonstrated that the mixture of another peptide amphiphile molecule with the same theoretical charge with ODN was able to form 1D nanofiber structures at 100:1 molar ratio of K-PA/ODN, as supported by zeta potential and polyacrylamide gel electrophoresis (PAGE) measurements. ${ }^{[11]}$ Self-assembly occurs between negatively charged ODN and positively charged lysine residues on peptide molecules. Therefore, the number of $\mathrm{CpG}$ ODNs bound to nanostructures is directly related with the B-PA to ODN ratio. As a result of PAGE analysis, we found that 50:1 molar ratio was critical for B-PA/ODN nanostructure, respectively (Figure S2, Supporting Information). In the light of previous results and Figure S2 (Supporting Information), the characterizations of nanofibrous complex and in vitro and in vivo experiments were performed with the nanofibrous complex prepared at 100:1 B-PA/ODN ratio. Secondary structure analyses of B-PA and B-PA/ODN nanostructure were assessed through circular dichroism (CD). The B-PA exhibited $\beta$-sheet formation, with a negative peak at $217 \mathrm{~nm}$ and a positive peak at $197 \mathrm{~nm}$ as a result of Val-Val-Ala peptide sequence. Similar results were observed in B-PA/ODN nanostructure following mixing B-PA with ODN, while no peak was present in the ODN-only (control) sample. CD results confirmed that B-PA formed $\beta$-sheets at physiological $\mathrm{pH}$ and that ODNs did not disrupt $\beta$-sheet formation (Figure 1B).

Scanning transmission electron microscopy (STEM) and atomic force microscopy (AFM) imaging were carried out to examine the morphological characteristics B-PA/ODN nanostructure in dried and aqueous environment, respectively (Figure 1D,C). STEM results show B-PA formed cylindrical nanostructures when mixed with ODN (Figure 1D). The 
A

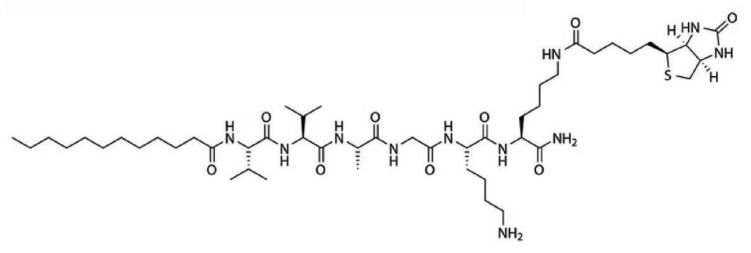

C

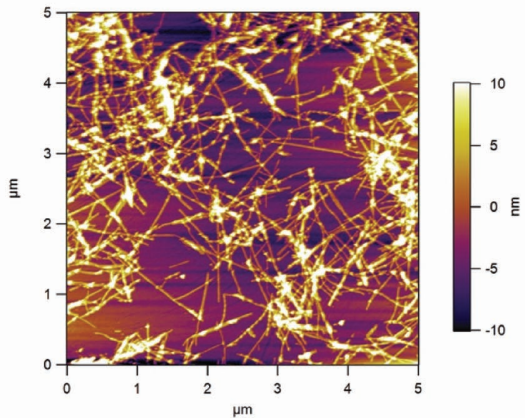

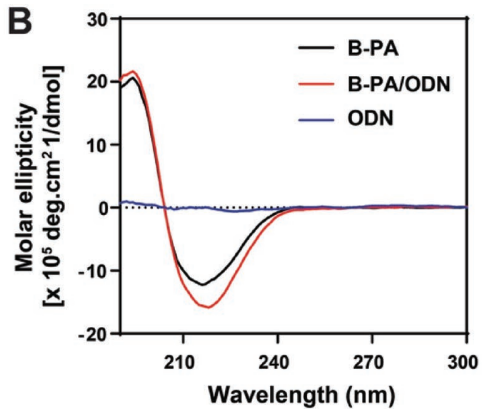

D

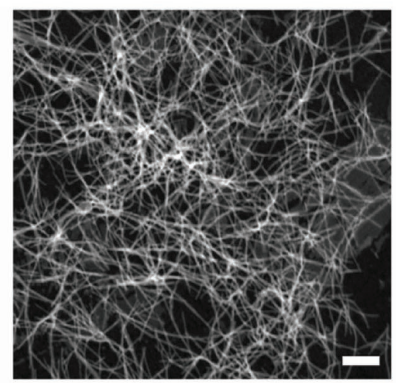

Figure 1. Structural characterization of PA/ODN nanostructures. A) Chemical representation of B-PA (Lauryl-VVAGKK (Bio)-Am). B) Circular dichroism (CD) spectra of ODN, B-PA, and B-PA/ODN nanostructures. C) AFM images of B-PA/ODN nanostructures in aqueous environment. D) STEM images of the B-PA/ODN nanostructures. Scale bar is $100 \mathrm{~nm}$.

presence of ODN appears to allow a complete self-assembly process, which is compatible with the CD, AFM, and STEM results (Figure 1B-D).

\subsection{Biotinylated Ovalbumin Binds to B-PA/ODN Nanofibers}

Ovalbumin (OVA) was chosen as the model antigen for the induction of nanofibrous complex mediated immune response due to its widespread use in immunology research. ${ }^{[20]}$ Streptavidin has four binding sites with exceptionally high affinity for biotin, allowing the use of streptavidin-biotin interactions for noncovalent antigen binding to the nanofibrous complex. ${ }^{[21]}$ Previous studies have demonstrated that OVA or OVA-derived sequences, which are covalently conjugated to designed materials can induce directed immune response. ${ }^{[14 \mathrm{~b}, 22]}$ The nanofibrous complex was prepared as follows: First, B-PA/ ODN nanofiber structures were obtained by mixing $0.023 \%$ (w/v) B-PA and $15 \mu \mathrm{g} \mathrm{mL} \mathrm{mL}^{-1}$ of ODN at 100:1 molar ratio, then, streptavidin was added to the mixture at the same molar ratio of B-PA. After 40 min incubation, biotinylated OVA was added to the mixture to obtain antigen bearing nanofiber structure.

To study the presence of OVA on nanofibrous complex, gold nanoparticle conjugated secondary antibody against anti-Ovalbumin (IgG-gold) was used. TEM imaging indicated the presence of OVA on nanofibrous complex (Figure 2A), however, when the primary antibody for OVA was not used no staining was observed (Figure 2B) indicating the specific binding of OVA to nanofibrous complex via streptavidin interaction. In addition to TEM imaging, the presence of OVA on nanofibrous complex was confirmed through emzyme-linked immunosorbent assay
(ELISA) analysis. PA/ODN nanofibers were obtained through self-assembly of ODN with the mixture of the biotinylated peptide amphiphile (B-PA) and non-biotinylated peptide amphiphile (K-PA) at different ratios. Therefore, biotin binding moieties on PA/ODN nanofibers were decreased which affects binding of OVA. As a result, in Figure S3A (Supporting Information), decreasing B-PA concentration in PA/ODN nanofibers resulted in decreased OVA binding. Although, decreasing biotin moieties on PA/ODN nanofibers directs first SA and then biotin-OVA binding, another experiment was performed to show whether all antigens attached to B-PA/ODN nanofibers or not. Figure S3B (Supporting Information) showed that not all of OVA antigens were attached to B-PA/ODN nanofibers. Thus, binding moieties on PA/ODN nanofibers are important for modulation of antigen amount on the nanostructures. Although free antigens in the delivery systems do not have negative effects on immunization, this will affect the uptake of adjuvant and antigen by same antigen presenting cells which might result in lower immune response.

\subsection{Nanofibrous Complex Elevates Cytokine Secretion}

IFN $\gamma$ and IL-12 are cytokines which are responsible for directing differentiation to the Th1 phenotype during the primary antigen response, and serve to build a bridge between innate and adaptive immune responses. ${ }^{[23]}$ IL-6, in contrast, known to promote Th2 immune response. ${ }^{[2]}$ ODN1826 was previously shown to promote IL-12, IFN $\gamma$, and IL- 6 secretion from splenocytes and also modulate the immune response toward Th1 phenotype. ${ }^{[25]}$ Immunomodulatory effects of nanofibrous complex were evaluated in vitro by using splenocytes. 

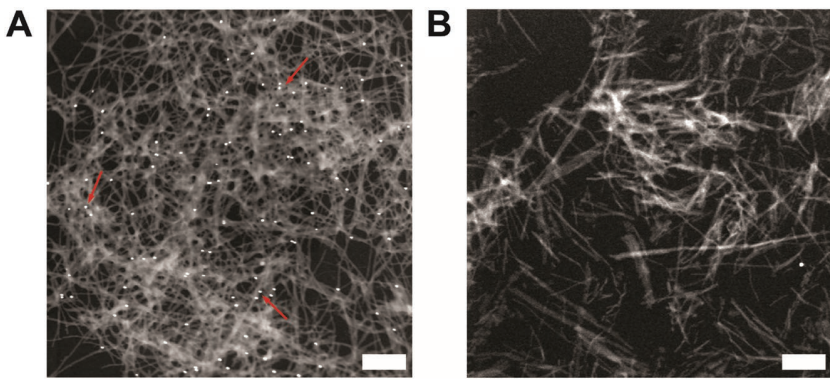

Figure 2. Antigen-bearing nanofibrous complex. A) STEM image of antiIgG-gold labeled nanofibrous complex. Scale bar is $500 \mathrm{~nm}$ (red arrows indicating gold labeling). B) STEM image of anti IgG-gold labeled nanofibrous complex without anti-Ovalbumin addition. Scale bar is $500 \mathrm{~nm}$.

The amount of cytokines in the culture supernatants were determined by ELISA. As shown in Figure 3, we found that nanofibrous complex dramatically promoted the expression of IFN $\gamma$, IL-12, and IL-6 compared to control group, results suggests that nanofibrous complex induce the secretion of IFN $\gamma$ and IL-12 from splenocytes, and a similar effect was also proposed as the potential mechanism-of-action for ODN1826. [25] Cytokine analysis showed that the nanofibrous complex protect the cytokine production feature of the ODN1826. In addition to cytokine secretion, enhanced IgG2a antibody response (Figure 5B), increased number of SIINFEKL-Kb positive (Figure 4D) and CD8+ T cells (Figure 6), the nanofibrous complex also promoted a more balanced Th1/Th2 response and altered immune response mostly toward Th1 phenotype. These results showed the importance of the close proximity between adjuvant and antigen and nanoformulated vaccine design.

\subsection{Nanofibrous Complex Enhances the Expression of Maturation Markers, Uptake, and Cross-Presentation of Antigens}

APCs, including dendritic cells (DCs), macrophages and B-cells, are specialized cells that capture and engulf exogenous antigens through endocytosis, digest them to isolate the epitopes they contain, and present these epitopes to CD4+ or CD8+ T cells through MHC class II or class I molecules, respectively. In addition to stimulation by $\mathrm{MHCs}$, the activation and proliferation of $\mathrm{T}$ cells also require the presence of costimulatory molecules such as CD40 and CD86. ${ }^{[26]}$ CD40 is expressed by APCs and serves as a costimulatory molecule, while TLR agonists assist in this process by increasing the expression levels of costimulatory molecules. Since ODN1826 functions as a TLR9 agonist, its ability to facilitate APC maturation in the presence and absence of a supporting peptide matrix was tested. ${ }^{[27]}$ In order to verify whether the nanofibrous complex has the capability of promoting APC maturation, splenocytes were treated with nanofibrous complex and control group for $24 \mathrm{~h}$. These combinations were administered as proof-of-concept doses for future in vivo experiments and to observe the behavior of the material in clinical concentrations. As shown in Figure 4A, nanofibrous complex significantly enhanced the expression of the both APC-stimulating marker CD86 and CD40 compared to control group (Figure 4B). These results show that nanofibrous complex is able to effectively promote APC maturation through the up-regulation of costimulatory molecules.

The effect of nanofibrous complex on ODN uptake was demonstrated on DC cells treated with nanofibrous complex (formed with flourescein isothiocyanate (FITC) labeled ODN) and control group (FITC labeled ODN + OVA) formulations for $2 \mathrm{~h}$. As shown in Figure 4C, uptake of nanofibrous complex was higher than the control group. Confocal images confirmed the uptake of both nanofibrous complex and control group (Figure S4, Supporting Information). However, sample preparation and variations make this method unfavorable for quantitative analysis. Cross-presentation involves the capture, endocytosis, and presentation of extracellular antigens to naive CD8+ T cells through MHC class I molecules. ${ }^{[28]}$ Splenocytes were treated with nanofibrous complex and control group to exhibit the efficacy of antigen-linked nanofibrous complex on antigen cross-presentation. Splenocytes were stained with an anti-H-2Kb/SIINFEKL monoclonal antibody that recognizes MHC I complex, and analyzed with flow cytometry. As shown in Figure 4D, nanofibrous complex enhanced OVA presentation significantly more than control group. This result shows that linking an antigen to a nanomaterial via noncovalent biotin-SA interaction is very efficient. Additionally, noncovalent binding
A

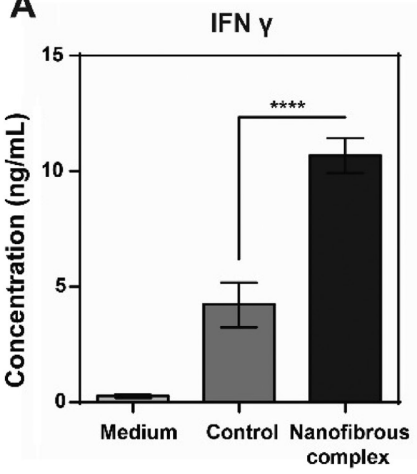

B

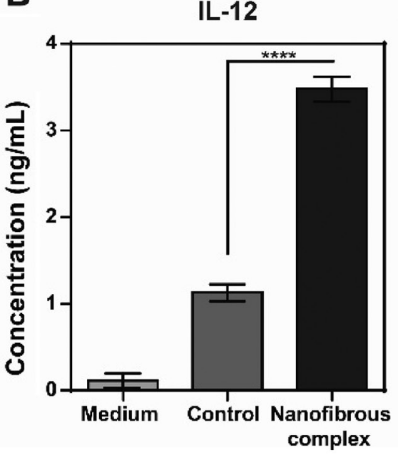

C

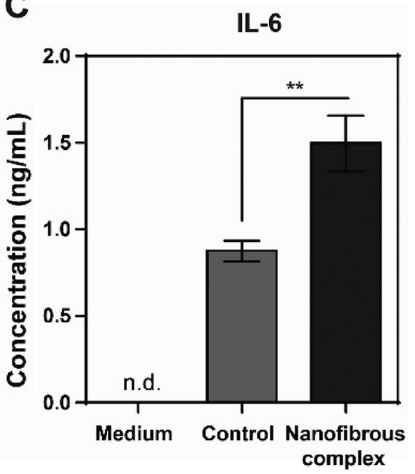

Figure 3. Cytokine secretion profile of splenocytes triggered by nanofibrous complex and control. Mouse splenocytes were treated with the indicated formulations, and cytokine concentrations in culture media were detected with ELISA: A) IFN $\gamma$, B) IL-12, and C) IL-6. ODN concentration in all groups

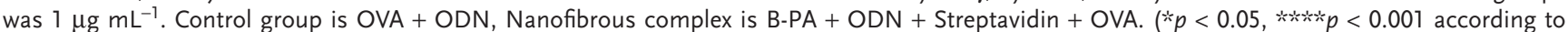
one-way ANOVA with Tukey's Multiple Comparison post-test, n.d. indicates "not detected"). 
A
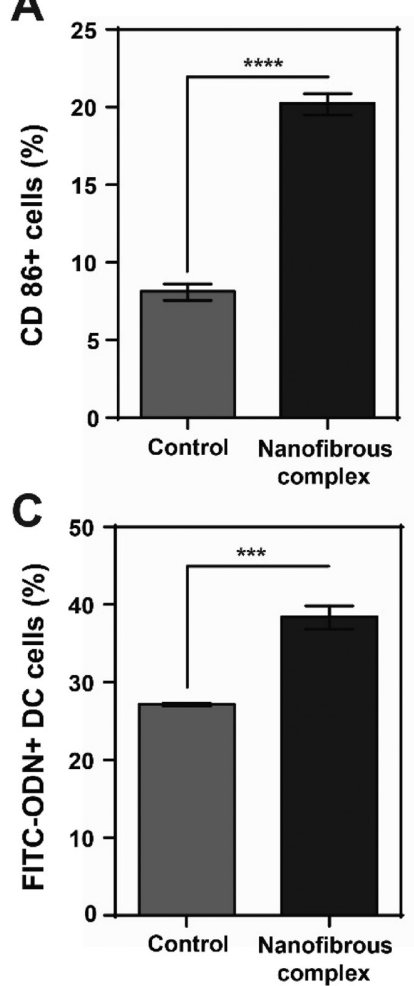

Figure 4. In vitro stimulation of immune responses. Flow-cytometry analysis of the expression of cell surface costimulatory markers. A) CD86 and B) CD40 on splenocytes. C) FITC-labeled CpG-ODN uptake by dendritic cells (CD11+ used for DCs number among splenocytes) ('**'* $p<0.001$ according to Student's $t$-test). D) Flow-cytometry analysis of cross-presentation of OVA on APC with anti-H-2Kb/SIINFEKL monoclonal antibody. Control group is OVA + ODN, Nanofibrous complex is B-PA + ODN + Streptavidin + OVA

of an antigen to a carrier vaccine system is more effective than soluble antigen administration. IFN $\gamma$ is known to be important for cross-presentation and the promotion of MHC class I molecules, ${ }^{[29]}$ and enhanced cytokine and IFN $\gamma$ secretion by nanofibrous complex could improve for cross-presentation of antigens (Figure 3). As a result, nanofibrous complex enhances uptake mechanism of ODN in DC cell, thus the antigen which facilitated SIINFEKL presentation.

\subsection{Effect of PA/ODN Nanostructures on Humoral Immunity and Cellular Immunity}

The immunogenicity of some antigens is limited, and it is especially problematic to produce effective vaccines incorporating these antigens. ${ }^{[3 \mathrm{a}]}$ As such, the potency of the nanofibrous complex as a vaccine system was studied in vivo to determine its ability to increase the immunogenicity of a model antigen OVA. In order to understand the effect of the nanofibrous complex on the immunogenicity of OVA, mice were immunized subcutaneously with different formulations of OVA twice at day 0 and day 15 . The antibody titer against antigen is an indicator for the evaluation of humoral immune responses. ${ }^{[30]}$ Consequently, blood was collected from animals at day 21 and OVA-specific IgG levels in sera were analyzed to determine the humoral immune response. Anti-OVA serum IgG titer was analyzed by ELISA (Figure S5, Supporting Information). Nanofibrous complex induced significantly higher anti-OVA IgG antibody production compared to control group (Figure 5A). Titration of IgG shows that level of IgG was so high in the nanofibrous complex that was detected at almost after 6000-fold dilution (Figure S5, Supporting Information). These results indicate that nanofibrous complex can strongly enhance the immune response in vivo, and that the observed effect is attributable to the close association between the antigen and nanofibrous complex.

The effect of nanofibrous complex on the relative frequency of IgG isotype was also tested, (Figure 5B). Interestingly, nanofibrous complex significantly enhanced IgG2a production compared to control group. It is well-recognized that IgG2a isotope is boosted by Th1 cytokines (such as IFN- $\gamma$ ). Therefore, it can be concluded that nanofibrous complex was able to enhance IgG2a isotype switching. Cytokine profiles of nanofibrous complex were also in good agreement with this result shown in Figure 3.

T-cell activation is important for vaccine performance, and CD8+ T cells are especially crucial for conditions such as intracellular infections and cancer. To promote the differentiation of functional $\mathrm{T}$ cells, the vaccine material should facilitate antigen entry into MHC class I or II processing pathways, trigger DC activation, and induce interferon (IFN) production. ${ }^{[36,31]}$ In order to assess the effect of nanofibrous complex on antigen specific $\mathrm{T}$ cells, spleens from immunized mice (1 week after second injection) were removed and the splenocytes were isolated. These splenocyte were restimulated with OVA. The IFN- $\gamma$ releasing profile and proliferation of restimulated cells were analyzed with ELISA and a standard BrdU assay, respectively. Supernatant of restimulated splenocyte were analyzed for IFN- $\gamma$ releasing. As shown in Figure 5C, the splenocytes of nanofibrous complex group secreted significantly more IFN- $\gamma$ than other groups. In addition, the nanofibrous complex significantly promoted splenocytes proliferation compared to control group when the splenocytes were restimulated with OVA. Moreover, proliferation was not observed in the groups when splenocytes were treated with culture medium (Figure 5D). These results showed that nanofibrous complex could significantly promote antigen-specific splenocyte proliferation and IFN- $\gamma$ release supporting that the proliferated cells are OVA specific cells.

To further investigate the effect of nanofibrous complex on the induction of OVA specific CD8+ T-cell formation, splenocytes of immunized mouse were isolated and restimulated with OVA. Extracellular and intracellular IFN $\gamma$ stainings of CD8+ T cells was performed after ex vivo restimulation of splenocytes with OVA in vitro (Figure 6A,B). The ratio of IFN- $\gamma+$ cells among CD8+ T cells was increased by nanofibrous complex more than the control group (Figure $6 \mathrm{C}$ ). These results are in good agreement with prior results obtained from SIINFEKL presentations (Figure 4D).

\section{Conclusions}

In this study, we report the first example of an antigen presenting cylindrical nanostructure complex that is formed 

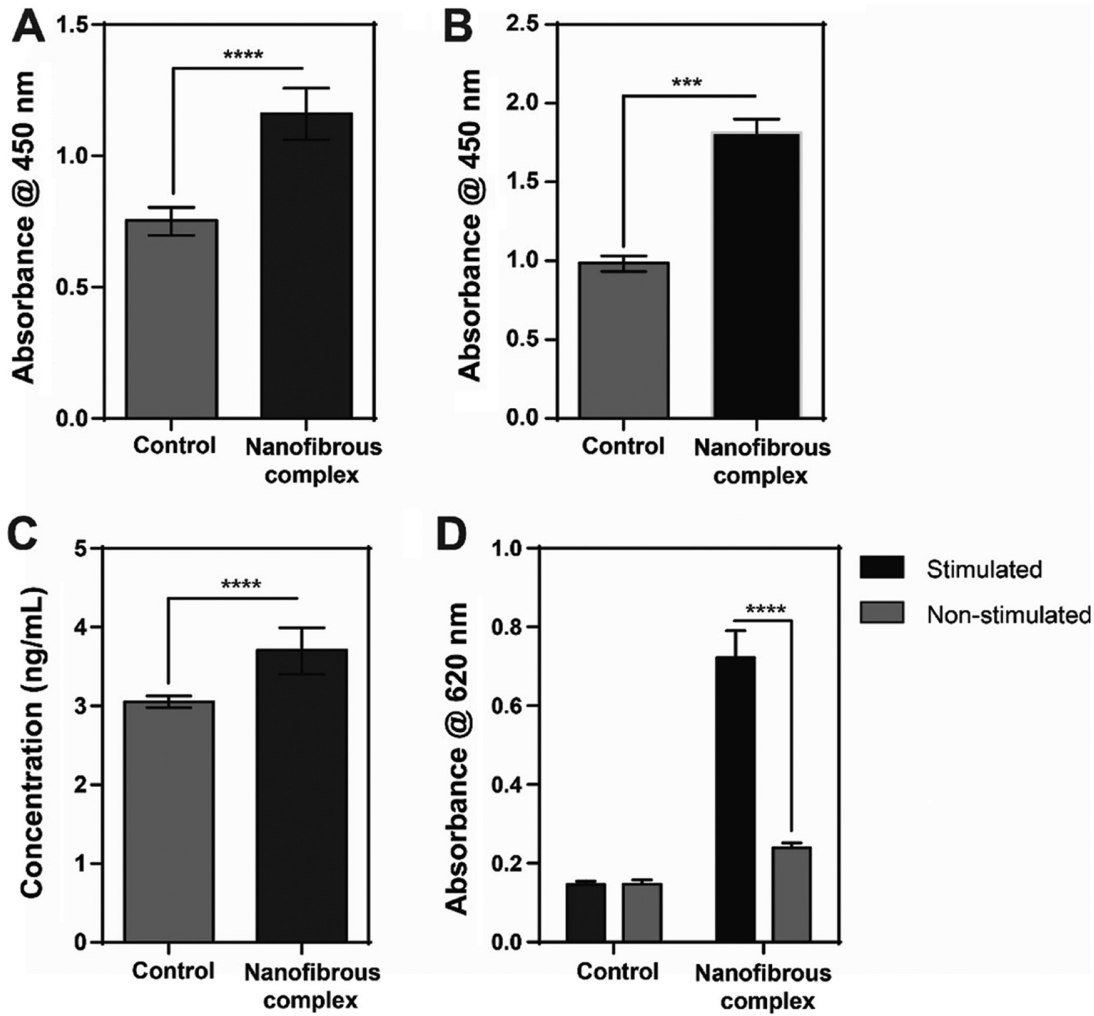

Figure 5. In vivo immunization with nanofibrous complex. $7 \mathrm{~d}$ after second injection, sera were isolated from mice and analyzed for IgG levels by ELISA (using tenfold diluted sera). A) OVA-specific antibody (IgG) production in sera from mice immunized with different groups, and B) IgG isotype IgG2a level in sera of immunized mice. The effect of nanofibrous complex and control on T-cell activation. C) IFN- $\gamma$ profile which was measured with ELISA after ex vivo restimulation of splenocytes with OVA. D) Splenocyte proliferation measured by BrdU assay. Control group is OVA + ODN, Nanofibrous complex is B-PA + ODN + Streptavidin + OVA $(* p<0.05$, **** $p<0.0001$ according to Student's $t$-test. Bars shown are mean \pm SEM $(n=3))$. shown via noncovalent biotin-streptavidin interaction as sandwich binding. We also demonstrated that the nanofibrous complex strongly induced antibody production and secretion of stimulatory cytokines, thereby directing the immune response toward the Th1 phenotype. Further investigations have revealed that immune induction by nanofibrous complex depends heavily on their ability to enhance antigen uptake, induce dendritic cell maturation, and enhance crosspresentation. Many of these properties are not present when the antigen is codelivered (but not covalently attached) with the nanocarrier systems, suggesting that the close proximity between the antigen and the adjuvant material played an important role in antigen presentation. The nanofibrous complex enables the presentation of viral, bacterial, or tumor antigens or antigenic peptide fragments on a single platform with adjuvant, enhances the immunogenicity of attached antigens, and promotes their cross-presentation, addressing many of the drawbacks associated with modern vaccine development. The streptavidin conjugated or biotinylated antigens or antigenic peptide fragments can be utilized for attachment to adjuvant carrier system with biotinylated nanofiber system, thus, makes this nanofiber structure a promising vaccine candidate for treatment of a broad variety of disorders and newly emerging diseases that necessitate the rapid development of vaccines. through the self-assembly of peptide amphiphile molecules with a pathogenic ODN and acts as an efficient vaccine for modulating both humoral and cellular immune responses in mice. We demonstrate that B-PA formed cylindrical nanostructures when coassembled with ODN. Antigen attachment was

\section{Experimental Section}

Materials: 9-Fluorenylmethoxycarbonyl (Fmoc) and tert-butoxycarbonyl (Boc) protected amino acids, [4-[ $\alpha$-(20,40-dimethoxyphenyl) Fmocaminomethyl] phenoxy] acetamidonorleucyl-4-methylbenzhydrilamine hydrochloride salt (MBHA) resin (Rink amide MBHA resin), and
A

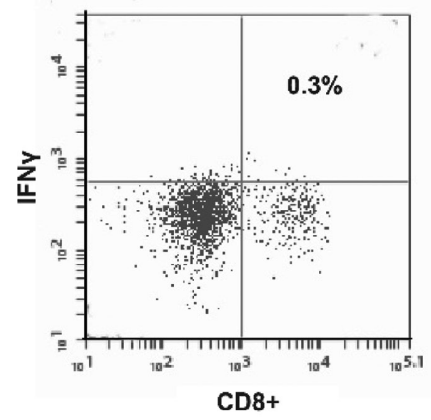

B

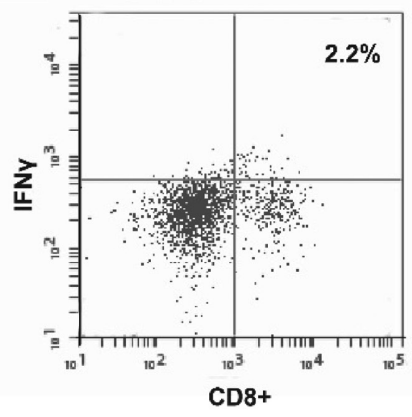

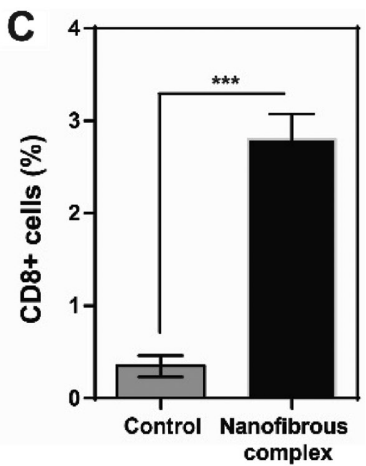

Figure 6. OVA specific $T$ cells in mice immunized with nanofibrous complex and control groups. A) ODN + OVA, B) B-PA/ODN+OVA, and C) cumulative bar graph from (A) and (B) showing the percentages of CD8+ T cells producing IFN- $\gamma$. OVA specific CD8+ T cells were detected by intracellular IFN-staining after ex vivo restimulation of immunized splenocytes with OVA in vitro. Control group is OVA + ODN, Nanofibrous complex is B-PA + ODN + Streptavidin + OVA ( $* \kappa^{-2} p<0.001$ according to Mann-Whitney. Bars shown are mean \pm SEM and $n=5$ ). 
Table 1. Used B-PA and ODN concentrations in polyacrylamide gel electrophoresis.

\begin{tabular}{lcc}
\hline Wells & Sample & Concentration \\
\hline 1 & 10 bp DNA ladder & \\
2 & Only ODN & $20 \mu \mathrm{g} \mathrm{mL}^{-1}$ ODN \\
3 & $1: 1$ & $0.00023 \%\left(3.06 \mu \mathrm{gL}^{-1}\right)$ B-PA: $20 \mu \mathrm{g} \mathrm{m}^{-1}$ ODN \\
4 & $10: 1$ & $0.0023 \%\left(30.6 \mu \mathrm{g} \mathrm{mL}^{-1}\right)$ B-PA: $20 \mu \mathrm{g} \mathrm{m}^{-1}$ ODN \\
5 & $50: 1$ & $0.0115 \%\left(153.3 \mu \mathrm{gL}^{-1}\right)$ B-PA: $20 \mu \mathrm{gL}^{-1}$ ODN \\
6 & $100: 1$ & $0.023 \%\left(306.6 \mu \mathrm{g} \mathrm{m}^{-1}\right)$ B-PA: $20 \mu \mathrm{gL} \mathrm{m}^{-1}$ ODN \\
7 & $250: 1$ & $0.0575 \%\left(766.6 \mu \mathrm{gL}^{-1}\right)$ B-PA: $20 \mu \mathrm{gL}^{-1}$ ODN \\
8 & Only B-PA & $0.023 \%(306.6 \mu \mathrm{g} \mathrm{mL}-1)$ B-PA \\
9 & 10 bp DNA ladder & \\
\hline
\end{tabular}

2-(1H-benzotriazol-1-yl)-1,1,3,3-tetramethyluronium hexafluorophosphate (HBTU) were purchased from NOVABiochem and ABCR. Other chemicals for peptide synthesis were purchased from Fisher, Merck, AlfaAesar, or Aldrich. CpG ODNs were purchased from Invivogen. Paired antibodies and recombinant proteins of IFN $\gamma$ and IL-12 were obtained from R\&D systems, that of IL-6 from eBioscience. Horseradish peroxidase-conjugated goat anti-mouse IgG (sigma), IgG1 (eBioscience), IgG2a (Abcam), or IgG2b (eBioscience) were obtained from Southern Biotechnologies (AL, USA). All cell culture and ELISA reagents were purchased from Life Technologies, except nonessential amino acid solution (Sigma-Aldrich). Fluorescently labeled antibodies were obtained from eBioscience (CD40, CD86, anti-mouse OVA257-264 (SIINFEKL) peptide).

Peptide Synthesis: Lauryl-VVAGKK(Biotin)-Am (B-PA) were synthesized on Rink amide MBHA resin. Amino acid couplings were performed with 2 equivalents (equiv) of Fmoc-protected amino acid, 1.95 equiv of $N, N, N^{\prime}, N^{\prime}$-tetramethyl-O-(1H-benzotriazol-1-yl)uronium hexafluorophosphate (HBTU) and 3 equiv of $N, N$-diisopropylethylamine for $2 \mathrm{~h}$. After remOVAl of Mtt group of Fmoc-Lys (Mtt)-OH by $5 \%$ trifluoroacetic acid (TFA) solution in dichloromethane (DCM), biotin was conjugated in the same manner as amino acid couplings. RemOVAl of Fmoc group was achieved by $20 \%$ (v/v) piperidine in dimethylformadide (DMF) for $20 \mathrm{~min}$. To block the remaining free amine groups after amino acid coupling, $10 \%(\mathrm{v} / \mathrm{v})$ acetic anhydride solution in DMF was used (30 min). After each step, the resin was washed using DMF, DCM, and DMF. A TFA/triisopropyl silane $/ \mathrm{H}_{2} \mathrm{O} / \mathrm{DCM}$ mixture (5:2.5:2.5:90 ratio) was used to cleave the peptide from the resins.

Preparation of Fiber Nanostructures: Nanofibers were prepared through the self-assembly of peptide molecules in the presence of oligonucleotides. In order to form fiber nanostructure, positively charged $0.023 \%(\mathrm{w} / \mathrm{v}) \mathrm{B}-\mathrm{PA}$ molecules were dissolved in $\mathrm{ddH}_{2} \mathrm{O}$ and mixed with $15 \mu \mathrm{g} \mathrm{mL} \mathrm{m}^{-1} \mathrm{CpC}$ (ODN1826) dissolved in $\mathrm{dd}_{2} \mathrm{O}$. The molar ratio for ensuring that all ODNs in solution interact with nanostructures was determined to be 100:1 for B-PA/ODN, as shown in our previous studies and PAGE. ${ }^{[1]}$ Nanofibrous complex was prepared at these ratios for all experiments throughout the experiments. Sequence of ODN1826 is $5^{\prime}$-tccatgacgttcctgacgtt- $3^{\prime}$. The ODN concentration was chosen according to previous studies, where it was shown that ODN1826 is more suitable for cytokines induction between 0.6 and $6 \mu \mathrm{g} \mathrm{mL}^{-1}$. ${ }^{[32]}$

Polyacrylamide Gel Electrophoresis: PAGE was performed to identify the critical B-PA/ODN ratio required to conjugate all ODNs in solution to PA nanostructures. In this experiment, ODN was used at $20 \mu \mathrm{g} \mathrm{mL}^{-1}$ concentration to make bands apparent in polyacrylamide gel. $15 \mu \mathrm{L}$ of $20 \mu \mathrm{g} \mathrm{mL} \mathrm{L}^{-1}$ ODN1826 solution was mixed with varying concentrations of $15 \mu \mathrm{L}$ of B-PA solutions (dissolved in nuclease-free water) to prepare different B-PA/ODN ratios (from 1:1 to 250:1) (Table 1). These solutions were mixed with Orange DNA loading dye (Fermentas) and loaded onto $20 \%$ polyacrylamide gels. $15 \mu \mathrm{L}$ of $10 \mathrm{bp}$ DNA ladder (O'range ruler, Fermentas) was used as marker. Gels were run at $75 \mathrm{~V}$ for $1 \mathrm{~h}$ and subsequently at $50 \mathrm{~V}$ for $2.5 \mathrm{~h}$ (in $1 \times$ Tris-acetate-EDTA (TAE)) at $4{ }^{\circ} \mathrm{C}$.
Stains-all dye working solution $(0.005 \%, \mathrm{w} / \mathrm{v})$ was prepared freshly from stock solution $(0.1 \% \mathrm{w} / \mathrm{v})$ as recommended by the manufacturer (SigmaAldrich). Gels were incubated in Stains-all overnight (dark conditions and $4{ }^{\circ} \mathrm{C}$ ). On the next day, the destaining of gels was performed under sunlight and images were taken by a Canon EOS Rebel T2i camera.

Preparation of Antigen Bearing Fiber Nanostructures: Nanofibrous complex was prepared by mixing B-PA, ODN, and streptavidin from Streptomyces avidinii (SA) (Sigma-Aldrich) and biotin-Ovalbumin (Galab). All components were dissolved in $d \mathrm{dH}_{2} \mathrm{O}$. First, nanofiber structure was established through self-assembly of positively charged $0.023 \%(\mathrm{w} / \mathrm{v})$ B-PA molecules with negatively charged $15 \mu \mathrm{g} \mathrm{mL}$ CpG (ODN1826). Then, streptavidin was mixed with B-PA/ODN nanofibers and incubated for $40 \mathrm{~min}$ and after that adding biotin-OVA to the mixture. The exact molar ratio for B-PA, streptavidin and biotinOvalbumin was 1:1:1. All preparations were made at room temperature.

Transmission Electron Microscopy: Nanostructures were imaged by TEM. $30 \mu \mathrm{L}$ of nanofibrous complex was prepared on parafilm by mixing $15 \mu \mathrm{L}$ of $15 \mu \mathrm{g} \mathrm{mL} \mathrm{L}^{-1}$ ODN1826 with $15 \mu \mathrm{L}$ of $0.023 \%$ (w/v) B-PA (100:1 ratio). TEM grids were inverted onto these solutions. Grids were removed after $10 \mathrm{~min}$ and the remaining solution on grid was absorbed by a lint-free paper. Staining was performed with $2 \%(\mathrm{w} / \mathrm{v})$ uranyl acetate solution (Ted Pella, Inc.) for $40 \mathrm{~s}$. Grids were washed with $\mathrm{dd}_{2} \mathrm{O}$ twice and dried for $3 \mathrm{~h}$ at room temperature. TEM imaging was performed by a FEI Tecnai G2F30 instrument. All images were taken in STEM mode with a high angle annular dark field (HAADF) detector.

Atomic Force Microscopy: AFM imaging of PA/ODN complexes was performed in liquid conditions. ODN1826 solution at $15 \mu \mathrm{g} \mathrm{mL} \mathrm{L}^{-1}$ concentration was mixed with an identical volume of $0.023 \%$ (w/v) B-PA solution (100:1 ratio). The final ODN concentrations in all groups were equal. Nanofibrous complex was diluted 10 times, and dropped onto the cleaved mica surface and imaged directly in aqueous environment (Figure 1D,F). Silicon tip $\left(150 \mathrm{kHz}, k=5 \mathrm{~N} \mathrm{~m}^{-1}\right)$ was used for tapping mode imaging of nanofibrous complex. MFP-3D Asylum microscope was used for imaging.

Circular Dichroism: CD spectroscopy was performed with a JASCO $1815 \mathrm{CD}$ spectrometer at room temperature. $0.2 \times 10^{-3} \mathrm{M}$ solutions of B-PA and mixtures with ODN1826 (100:1) were measured from 300 to $190 \mathrm{~nm}$. Scanning speed, data pitch, data integration time (DIT), and bandwidth were adjusted to $100 \mathrm{~nm} \mathrm{~min}-1,1 \mathrm{~nm}, 4 \mathrm{~s}$, and $1 \mathrm{~nm}$, respectively. All measurements were performed with three data accumulations and sensitivity was selected as standard.

Immunogold Staining and Transmission Electron Microscopy: $30 \mu \mathrm{L}$ of nanofibrous complex was prepared on parafilm by mixing $15 \mu \mathrm{L}$ of $0.023 \%(\mathrm{w} / \mathrm{v}) \mathrm{B}-\mathrm{PA}$ and $15 \mu \mathrm{L}$ of $15 \mu \mathrm{g} \mathrm{mL}-1$ ODN1826 with (molar 100:1 ratio). TEM grids were inverted onto these solutions. Grids were removed after $10 \mathrm{~min}$ and the remaining solution on grid was absorbed by a lint-free paper. Grids were reversed onto $30 \mu \mathrm{L}$ of blocking solution (Assay buffer, Invitrogen) and incubated for $2 \mathrm{~h}$ at room temperature. Drops on grids were absorbed, and streptavidin $\left(915 \mathrm{ng} \mathrm{mL}^{-1}\right.$ in double-distilled water $\left(\mathrm{ddH}_{2} \mathrm{O}\right)$ ) was added onto the grids. After $1 \mathrm{~h}$ of incubation, grids were washed five times with $\mathrm{ddH}_{2} \mathrm{O}$. Drops on grids were absorbed, and OVA $\left(685 \mathrm{ng} \mathrm{mL}^{-1}\right.$ in $\left.\mathrm{dd}_{2} \mathrm{O}\right)$ was added onto the grids and incubated for $1 \mathrm{~h}$ at room temperature. Primary antibody $\left(0.25 \mu \mathrm{g} \mathrm{mL} \mathrm{L}^{-1}, \mathrm{Abcam}\right)$ against mouse OVA was added onto grids and incubated overnight at $4{ }^{\circ} \mathrm{C}$. Grids were washed with $\mathrm{ddH}_{2} \mathrm{O} 5$ times. Gold-conjugated antibody ( $25 \mathrm{~nm}$ gold particles conjugated to antimouse $\operatorname{lgG}$ ), $1 / 20$ diluted from stock with assay buffer, was put onto paraffin film, and grids were reversed onto this solution. After $1 \mathrm{~h}$, grids were washed five times with $\mathrm{dd}_{2} \mathrm{O}$. After drying at room temperature for at least $3 \mathrm{~h}$, TEM (FEl, Tecnai G2 F30) imaging was performed. All images were taken in STEM mode with an HAADF detector.

ELISA Assay for Ovalbumin Binding to PA/ODN Nanofibers: For the first experiment, PA/ODN nanostructure was formed by mixing $20 \mu \mathrm{L}$ of $15 \mu \mathrm{g} \mathrm{mL} \mathrm{m}^{-1}$ ODN 1826 with $20 \mu \mathrm{L}$ of either $0.023 \%$ (w/v) B-PA $(100: 1$ ratio), $0.015 \%(\mathrm{w} / \mathrm{v})$ K-PA (100:1 ratio) or different ratios of K-PA/B-PA mixtures (Figure S3A, Supporting Information). Then, either B-PA/ ODN nanofibers or K-PA/B-PA mixtures were added to ELISA plates. For, the second experiment, B-PA/ODN nanofibers was formed by 
mixing $20 \mu \mathrm{L}$ of $15 \mu \mathrm{g} \mathrm{mL} \mathrm{L}^{-1}$ ODN1826 with $20 \mu \mathrm{L}$ of either $0.023 \%$ (w/v) B-PA (100:1 ratio) (Figure S3B, Supporting Information). Then, both B-PA/ODN nanofibers or only Ovalbumin (OVA) antigen were added to ELISA plates. The remaining part of the experiments is same and performed as following: The next day, the solutions were removed and the wells were washed with washing buffer (Tween 20 in $0.9 \%$ $(\mathrm{w} / \mathrm{v}) \mathrm{NaCl}$ solution, $\mathrm{pH}=7.4)$. Then, plates were dried by tapping and blocked with $0.5 \%$ bovine serum albumin (BSA) ( $2 \mathrm{~h}$ ). After that, antigen attachment to the nanofibers was achieved by incubation first with SA and then OVA for $1 \mathrm{~h}$. Plates were washed 5 times with washing buffer and dried by tapping between each consecutive step. Primary antibody $\left(0.25 \mu \mathrm{g} \mathrm{mL} \mathrm{m}^{-1}, \mathrm{Abcam}\right)$ against mouse OVA was added. Plate was incubated overnight at $4{ }^{\circ} \mathrm{C}$. On the next day, plates were washed 5 times with washing buffer and dried by tapping and adding IgG-HRP (horse radish peroxidase)-conjugated IgC $(2 \mathrm{~h})$, consecutively, at room temperature. Plates were washed 5 times with washing buffer and dried by tapping in between. 3,3',5,5'-Tetramethylbenzidine (TMB) substrate was added at the last step and reaction was stopped after 30 min by $1.8 \mathrm{~N} \mathrm{H}_{2} \mathrm{SO}_{4}$. Color formation was measured by microplate reader (Spectramax M5, Molecular Devices) as absorbance at $450 \mathrm{~nm}$ wavelength. In order to obtain exact absorbance values solely to dye color, the value was subtracted from a reference value $(650 \mathrm{~nm})$.

Animal Models: All experimental procedures involving animals were approved and performed according to the Animal Ethics Committee of Gazi University Hospital (Protocol G.Ü.ET-15.063). Primary spleen cells were obtained from adult BALB/C and C57BL/6 (10-14 weeks old) mice, which were maintained under controlled conditions and fed ad libitum.

Maintenance of Splenocytes and Stimulation Experiments: Spleens were removed and single cells were dissociated from bulk tissue by using the end of a syringe in culture media (2\% FBS in RPMI-1640). Single cell suspension was collected carefully to exclude tissue debris. Cell suspension was centrifuged at $800 \mathrm{~g}$ for $10 \mathrm{~min}$. Supernatant was discarded and cell pellet was resuspended in $1 \mathrm{~mL}$ red blood cell lysis buffer (10x RBC lysis buffer: $0.155 \mathrm{M} \mathrm{NH}_{4} \mathrm{Cl} ; 0.01 \mathrm{M} \mathrm{KHCO}_{3} ; 0.1 \times 10^{-3} \mathrm{M}$ EDTA dissolved in $1 \mathrm{~L}$ of $d d \mathrm{H}_{2} \mathrm{O}$ and diluted to $1 \times \mathrm{RBC}$ lysis buffer). Then, $9 \mathrm{~mL}$ of RPMI-1640 with 5\% FBS was added and centrifuged at $800 \mathrm{~g}$ for $10 \mathrm{~min}$ (these steps were performed three times). Cells were adjusted to $2 \times 10^{6}$ cells $\mathrm{mL}^{-1}$ cell density and cultured in 96-well plates as $200 \mu \mathrm{L}$ per well $\left(5 \times 10^{5}\right.$ cells per well). The medium used for the splenocyte culture was composed of RPMI-1640 with 5\% FBS (1\% Pen/Strep, $1 \%$ L-Glu, $1 \%$ nonessential amino acids and HEPES $\left(20 \times 10^{-3} \mathrm{M}\right)$ were also added). Cell stimulation was performed immediately after distributing cells to wells. Nanofibrous complex was prepared as described previously. Nanofibrous complex and control group solutions were further diluted with media and final concentration of ODN in cell suspension was $1 \mu \mathrm{g} \mathrm{mL} \mathrm{m}^{-1}$. For cytokine analysis, cells were cultured at $37{ }^{\circ} \mathrm{C}$ and $5 \% \mathrm{CO}_{2}$ for $48 \mathrm{~h}$ and supernatants were collected at the end of the experiment. For the analysis of surface markers (costimulatory molecules), SIINFEKL presenting cells were treated with the same formulations for $24 \mathrm{~h}$. Cells were collected at the end of the experiment for further staining and analysis by flow cytometry. All experiments were performed in triplicate; representative results of three independent experiments are shown.

ELISA: Effect of nanofibrous complex on splenocytes was checked by measuring cytokine concentrations in supernatants collected from stimulation experiment with ELISA. MaxiSorpTM plates (Thermo Scientific, NUNC) were coated with IL-6, IL-12, or IFN- $\gamma$ primary antibodies (overnight incubation at $4{ }^{\circ} \mathrm{C}$ ). The next day, plates were blocked with $0.5 \%$ BSA $(2 \mathrm{~h})$, incubated with supernatants of cell culture experiment or standard recombinant proteins $(24 \mathrm{~h})$, biotinlabeled secondary antibody $(2 \mathrm{~h})$, and horse radish peroxidase (HRP)conjugated streptavidin $(1 \mathrm{~h})$, consecutively, at room temperature. Plates were washed five times with washing buffer and dried by tapping between each consecutive step. TMB substrate was added at the last step and reaction was stopped after 15-20 min by adding $1.8 \mathrm{~N} \mathrm{H}_{2} \mathrm{SO}_{4}$. Color formation was measured by microplate reader (Spectramax M5, Molecular Devices) as absorbance at $450 \mathrm{~nm}$ wavelength (with a reference value at $650 \mathrm{~nm}$ ). All treatments were performed with at least three replicates and are shown as mean $+/-$ standard deviation.

Flow Cytometry Analyses of Surface Markers and SIINFEKL: For analysis of the expression of costimulatory molecules and SIINFEKL presentation, cells were stained with CD40 and CD86 and SIINFEKL antibodies. Treated cells were collected and washed with $1 \times$ phosphate buffered saline (PBS), centrifuged and resuspended in FACS buffer ( $3 \%$ BSA $+1 \%$ sodium azide in $1 \times$ PBS). Flow cytometry was performed with BD Accuri C6-Cytometer equipment with BD Accuri C6 Software. The number of events was at least 20000 for all samples. The experiment was performed in triplicate and representative results of two independent experiments are shown.

Evaluation of Effects of Nanofibrous Complex on Immunized Mice: For in vivo immune evaluation, male 10-14 weeks old Balb/C mice were administered with $500 \mu \mathrm{L}$ of vaccine formulations subcutaneously (s.c.). 4 groups $(n=5)$ of animals were treated with OVA (antigen) alone, OVA with CPG ODN, and nanofibrous complex (all in isotonic sucrose solution). $10 \mu \mathrm{g}$ of OVA was given to all animals, while CpG ODN amounts were $10 \mu \mathrm{g}$ in relevant groups. Booster injections were performed on day 15. At days 13 and 28 , animals were bled, and sera were obtained. IgG amounts in sera were detected with ELISA. OVA antigen was coated onto 96-well plates, blocked with $1 \%$ BSA buffer, and serially diluted (tenfold) sera were added onto the wells. IgG was detected with HRP conjugated anti-lgG. Absorbance in each well was measured after TMB substrate addition. For analyzing splenocyte proliferation, animals were sacrificed at days 21 and 28 and spleens were removed. Single cells were dissociated from bulk tissue and $5 \times 10^{5}$ splenocytes per well were seeded to 96 well plates, and then BrdU assay was performed.

Effects of Nanofibrous Complex on Splenocyte Proliferation: Proliferation of cells after OVA stimulation was assessed through BrdU assay (Roche). Cells were seeded into wells at a density of $5 \times 10^{5}$ cells per well. Cells were incubated in standard cell culture medium supplemented with $10 \times 10^{-6} \mathrm{M}$ BrdU labeling solution for $24 \mathrm{~h}$. At the end of the incubation, BrdU incorporation assay was performed according to the manufacturer's instructions. Briefly, cells were fixed with FixDenat for $30 \mathrm{~min}$ and anti-BrdU-POD solution was added into wells. Following 90 min of incubation and tapping, substrate solution was added into wells and proliferation rates of the cells were quantified by measuring the absorbance (370 nm, with $492 \mathrm{~nm}$ reference wavelength) with microplate reader.

Uptake of Nanofibrous Complex into Splenocytes: Internalization of ODNs and nanofibrous complex into antigen presenting cells (APCs), which were freshly isolated from spleen, was analyzed by flow cytometry. For this purpose, FITC-conjugated ODN was used for preparing nanofibrous complex. Mouse splenocytes were seed in 96-well plates $\left(5 \times 10^{5}\right.$ cells per well). Cells were treated with nanofibrous complex and control group, or each group with OVA was incubated for $2 \mathrm{~h}$ before flow cytometry experiment. Cells were collected into $1.5 \mathrm{~mL}$ Eppendorf tubes by pipetting, and centrifuged. Supernatants were discarded, cells were washed with $1 \times$ PBS and a cell pellet was obtained again by centrifugation for further staining with specific antibody and analyses by flow cytometry. Flow cytometry was performed with BD Accuri C6-Cytometer equipment with BD Accuri C6 Software. The number of events was at least 20000 for all samples.

Uptake of Nanofibrous Complex into Immature Dendritic Cells: $9 \mathrm{~mm}$ glass coverslips were placed in 48-well plates and $2.5 \times 10^{4}$ THP-1 human monocyte cells were seeded in RPMI medium containing $10 \%$ FBS, rhIL-4 (100 ng mL $\mathrm{m}^{-1}$ ) and rhGM-CSF $\left(100 \mathrm{ng} \mathrm{mL}^{-1}\right)$. Cells were cultured for $5 \mathrm{~d}$ to obtain immature DCs. Medium was exchanged every $2 \mathrm{~d}$ with fresh cytokine-supplemented medium. At the end of the 5th day, Nanofibrous complex or control group were administered and cells were incubated for $2 \mathrm{~h}$. After incubation, cells were washed with fresh medium and PBS three times, fixed with $4 \%$ paraformaldehyde for $15 \mathrm{~min}$ at room temperature. Then, the cells were permeabilized with $0.1 \%$ Triton-X and blocked with $3 \%$ BSA in PBS solution for $2 \mathrm{~h}$. The stainings with phalloidin-TRITC (1:500) and TO-PRO-3 (1:1000) were performed for $20 \mathrm{~min}$ in $1 \times$ PBS. After serial washing with $1 \times$ PBS for 
10 min each, coverslips were mounted with ProLong Gold Antifade Reagent (Thermofisher). Cells were visualized by using Laser Scanning Confocal Microscope (LSM 510, Zeiss) at 20× magnification.

\section{Supporting Information}

Supporting Information is available from the Wiley Online Library or from the author.

\section{Acknowledgements}

The authors would like to thank M. Guler for technical help in TEM imaging. The authors thank A. D. Ozkan for fruitful discussions. S.T. was supported by TUBITAK BIDEB-2210 M.Sc. fellowship. N.G., M.A.K., and A.E.T were supported by a TUBITAK BIDEB-2211 Ph.D. fellowship. This work was partially supported by the Scientific and Technological Research Council of Turkey (TUBITAK 114Z562) and the TUBA.

\section{Conflict of Interest}

The authors declare no conflict of interest.

\section{Keywords}

adaptive immunity, antigen-presenting nanostructures, CD8+ T-cell response, peptide amphiphile nanofibers, vaccine development

Received: January 19, 2017

Revised: March 20, 2017

Published online: May 2, 2017

[1] a) G. J. Nabel, N. Engl. J. Med. 2013, 368, 551; b) D. Wu, Y. Gao, Y. Qi, L. Chen, Y. Ma, Y. Li, Cancer Lett. 2014, 351, 13.

[2] J. Conniot, J. M. Silva, J. G. Fernandes, L. C. Silva, R. Gaspar S. Brocchini, H. F. Florindo, T. S. Barata, Front. Chem. 2014, 2, 105.

[3] a) N. Petrovsky, J. C. Aguilar, Immunol. Cell Biol. 2004, 82, 488 b) R. L. Coffman, A. Sher, R. A. Seder, Immunity 2010, 33, 492.

[4] a) B. D. Walker, D. R. Burton, Science 2008, 320, 760; b) S. G. Reed S. Bertholet, R. N. Coler, M. Friede, Trends Immunol. 2009, 30, 23.

[5] N. Zhang, R. Channappanavar, C. Ma, L. Wang, J. Tang, T. Garron, X. Tao, S. Tasneem, L. Lu, C.-T. K. Tseng, Cell. Mol. Immunol. 2016 $13,180$.

[6] A. Bråve, K. Ljungberg, B. Wahren, M. A. Liu, Mol. Pharm. 2007, 4, 18.

[7] a) C. Foged, Ther. Delivery 2011, 2, 1057; b) J. J. Moon, H. Suh A. Bershteyn, M. T. Stephan, H. Liu, B. Huang, M. Sohail, S. Luo,
S. H. Um, H. Khant, J. T. Goodwin, J. Ramos, W. Chiu, D. J. Irvine, Nat. Mater. 2011, 10, 243.

[8] P. Couvreur, C. Vauthier, Pharm. Res. 2006, 23, 1417.

[9] T. Uto, T. Akagi, T. Hamasaki, M. Akashi, M. Baba, Immunol. Lett. 2009, 125, 46

[10] a) L. E. Guimarães, B. Baker, C. Perricone, Y. Shoenfeld, Pharmacol. Res. 2015, 100, 190; b) R. Edelman, Mol. Biotechnol. 2002, 21, 129.

[11] R. Mammadov, G. Cinar, N. Gunduz, M. Goktas, H. Kayhan, S. Tohumeken, A. E. Topal, I. Orujalipoor, T. Delibasi, A. Dana, S. Ide, A. B. Tekinay, M. O. Guler, Sci. Rep. 2015, 5, 16728

[12] M. F. Bachmann, G. T. Jennings, Nat. Rev. Immunol. 2010, 10, 787.

[13] A. Jegerlehner, T. Storni, G. Lipowsky, M. Schmid, P. Pumpens, M. F. Bachmann, Eur. J. Immunol. 2002, 32, 3305.

[14] a) G. A. Hudalla, T. Sun, J. Z. Gasiorowski, H. Han, Y. F. Tian, A. S. Chong, J. H. Collier, Nat. Mater. 2014, 13, 829; b) M. Black, A. Trent, Y. Kostenko, J. S. Lee, C. Olive, M. Tirrell, Adv. Mater. 2012, 24, 3845; c) H. Cui, M. J. Webber, S. I. Stupp, Biopolymers 2010, 94 , 1; d) J. S. Rudra, T. Sun, K. C. Bird, M. D. Daniels, J. Z. Gasiorowski, A. S. Chong, J. H. Collier, ACS Nano 2012, 6, 1557.

[15] a) G. A. Hudalla, J. A. Modica, Y. F. Tian, J. S. Rudra, A. S. Chong, T. Sun, M. Mrksich, J. H. Collier, Adv Healthcare Mater. 2013, 2, 1114; b) J. S. Rudra, Y. F. Tian, J. P. Jung, J. H. Collier, Proc. Natl. Acad. Sci. USA 2010, 107, 622.

[16] A. Trent, B. D. Ulery, M. J. Black, J. C. Barrett, S. Liang, Y. Kostenko, N. A. David, M. V. Tirrell, AAPS J. 2015, 17, 380.

[17] A. Holmberg, A. Blomstergren, O. Nord, M. Lukacs, J. Lundeberg M. Uhlén, Electrophoresis 2005, 26, 501.

[18] M. E. Davis, P. C. Hsieh, T. Takahashi, Q. Song, S. Zhang, R. D. Kamm, A. J. Grodzinsky, P. Anversa, R. T. Lee, Proc. Natl. Acad. Sci. USA 2006, 103, 8155.

[19] D. Mumcuoglu, M. Sardan Ekiz, G. Gunay, T. Tekinay, A. B. Tekinay, M. O. Guler, ACS Appl. Mater. Interfaces 2016, 8, 11280.

[20] H. X. Sun, Y. P. Ye, H. J. Pan, Y. J. Pan, Vaccine 2004, 22, 3882.

[21] P. S. Stayton, T. Shimoboji, C. Long, A. Chilkoti, G. Chen, I. M. Harris, A. S. Hoffman, Nature 1995, 378, 472.

[22] E. M. Bachelder, T. T. Beaudette, K. E. Broaders, S. E. Paramonov, J. Dashe, J. M. Fréchet, Mol. Pharm. 2008, 5, 876

[23] T. F. Gajewski, H. Schreiber, Y. X. Fu, Nat. Immunol. 2013, 14, 1014

[24] S. Diehl, M. Rincón, Mol. Immunol. 2002, 39, 531.

[25] P. S. Walker, T. Scharton-Kersten, A. M. Krieg, L. Love-Homan, E. D. Rowton, M. C. Udey, J. C. Vogel, Proc. Natl. Acad. Sci. USA 1999, 96, 6970.

[26] L. Chen, Nat. Rev. Immunol. 2004, 4, 336.

[27] A. M. Krieg, Oncogene 2008, 27, 161.

[28] W. R. Heath, F. R. Carbone, Nat. Rev. Immunol. 2001, 1, 126.

[29] K. Schroder, P. J. Hertzog, T. Ravasi, D. A. Hume, J. Leukocyte Biol. 2004, 75, 163

[30] H. Wang, Z. Luo, Y. Wang, T. He, C. Yang, C. Ren, L. Ma, C. Gong, X. Li, Z. Yang, Adv. Funct. Mater. 2016, 26, 1822.

[31] J. J. Engelhardt, B. Boldajipour, P. Beemiller, P. Pandurangi, C. Sorensen, Z. Werb, M. Egeblad, M. F. Krummel, Cancer Cell 2012, 21, 402.

[32] P. S. Walker, T. Scharton-Kersten, A. M. Krieg, L. Love-Homan, E. D. Rowton, M. C. Udey, J. C. Vogel, Proc. Natl. Acad. Sci. USA 1999, 96, 6970. 